\title{
The existence and stability for weakly Ky Fan's points of set-valued mappings
}

Wensheng Jia ${ }^{1,2}$, Shuwen Xiang ${ }^{2 *}$, Jihao $\mathrm{He}^{2}$ and Yanlong Yang ${ }^{3}$

"Correspondence: swxiang@163.com

${ }^{2}$ College of Science, Guizhou University, Guiyang, Guizhou 550025, P.R. China

Full list of author information is available at the end of the article

\begin{abstract}
In this paper, the notion of weakly Ky Fan's points of set-valued mappings is established, and we prove some existence theorems of weakly Ky Fan's points for functions with no continuity or space with no compactness. Then, from the viewpoint of the essential stability, we prove that most of problems in weakly Ky Fan's points (in the sense of Baire category) are essential.
\end{abstract}

MSC: 26D20; 26E25

Keywords: weakly Ky Fan's points; set-valued mappings; C-concave; C-quasiconcave-like; essential solution

\section{Introduction}

Ky Fan [1] gave an inequality for real valued functions which plays a very important role in nonlinear analysis (e.g., see Lin and Simons [2]). Let $X$ be a nonempty compact convex subset of a Hausdorff topological vector space, and $\varphi: X \times X \rightarrow \Re$ be such that (1) $\varphi(x, x) \leq$ 0 for all $x \in X$; (2) for each fixed $x \in X, y \rightarrow \varphi(x, y)$ is lower semicontinuous; (3) for each fixed $y \in X, x \rightarrow \varphi(x, y)$ is quasiconcave, then there exists $y^{*} \in X$ such that $\varphi\left(x, y^{*}\right) \leq 0$ for all $x \in X$.

Tan, Yu and Yuan [3] defined the inequality above as the Ky Fan inequality and called such a point $y^{*}$ Ky Fan's point, which is fundamental in proving many theorems in nonlinear analysis such as optimization problem, Nash equilibrium problem, variational inequality problem. There have been numerous generalizations of the Ky Fan inequality (see [4-8]). In [4], Yu and Yuan studied the existence of weight Nash equilibria and Pareto equilibria for multiobjective games using the Ky Fan minimax inequality. In [5], Luo proved the existence of an essential component of the solution set for vector equilibrium problems. Yang and $\mathrm{Yu}$ [6] gave a generalization of the Ky Fan inequality to vector-valued functions. They proved that for every vector-valued function (satisfying some continuity and convexity condition), there exists at least one essential component of the set of its Ky Fan's points. Yu and Xiang [8] proposed a notion of essential components of Ky Fan's points and proved its existence under some conditions, the Ky Fan's points have at least one essential component. Besides, they proved that for every $n$-persons noncooperative game, there exists at least one essential component of the set of its Nash equilibrium points. Zhou, Xiang and Yang [9] studied the stability of solutions for Ky Fan's section theorem with some applications. For our purpose, we give the notion of weakly Ky Fan's points of set-valued mappings and obtain some existence theorems of weakly Ky Fan's points for functions with no continuity or space with no compactness. Then, we prove that most of 
problems in weakly Ky Fan's points (in the sense of Baire category) are essential, thus they are stable. Our results include corresponding results in the literature as a special case.

\section{Preliminaries}

Now we recall some definitions in $[10,11]$.

Definition 2.1 Let $X$ and $Y$ be two Hausdorff topological spaces, and $F: X \rightarrow 2^{Y}$ be a set-valued mapping.

(1) $F$ is said to be upper semicontinuous at $x \in X$, if for any open subset $O$ of $Y$ with $O \supset$ $F(x)$, there exists an open neighborhood $U(x)$ of $x$ such that $O \supset F\left(x^{\prime}\right)$ for any $x^{\prime} \in U(x)$ and $F$ is said to be upper semicontinuous on $X$, if $F$ is upper semicontinuous at each $x \in X$.

(2) $F$ is said to be lower semicontinuous at $x \in X$, if for any open subset $O$ of $Y$ with $O \cap F(x) \neq \emptyset$, there exists an open neighborhood $U(x)$ of $x$ such that $O \cap F\left(x^{\prime}\right) \neq \emptyset$ for any $x^{\prime} \in U(x)$ and $F$ is said to be lower semicontinuous on $X$, if $F$ is lower semicontinuous at each $x \in X$.

(3) $F$ is said to be a usco mapping, if $F$ is upper semicontinuous on $X$ and $F(x)$ is compact for each $x \in X$.

(4) $F$ is said to be closed, if $\operatorname{Graph}(F)=\{(x, y) \in X \times Y \mid y \in F(x)\}$ is closed.

Definition 2.2 Let $H$ be a topological vector space and $C$ be a cone of $H$. A cone $C$ is said to be convex, if $C+C=C$, and a cone $C$ is said to be pointed, if $C \cap C=\{\theta\}$, where $\{\theta\}$ denotes the zero element of $H$.

Remark 2.3 (see [6]) If $C$ is a closed, convex, pointed cone with int $C \neq \emptyset$, where int $C$ denotes the interior of $C$ in $H$, then we can easily obtain that int $C+C=\operatorname{int} C$.

Definition 2.4 Let $X$ and $Y$ be two topological vector spaces, $K$ be a nonempty convex subset of $X, F: K \rightarrow 2^{Y}$ be a set-valued mapping, and $C$ be a closed, convex, pointed cone with int $C \neq \emptyset$.

(1) $F$ is said to be $C$-concave, if for every $x_{1}, \ldots, x_{n} \in K$ and $\lambda_{i} \in[0,1], \sum_{i=1}^{n} \lambda_{i}=1$ then $F\left(\sum_{i=1}^{n} \lambda_{i} x_{i}\right) \subset \sum_{i=1}^{n} \lambda_{i} F\left(x_{i}\right)+C$ and $C$-convex if $-F$ is $C$-concave.

(2) $F$ is said to be $C$-quasiconcave-like, if for every $x_{1}, \ldots, x_{n} \in K$ and $\lambda_{i} \in[0,1], \sum_{i=1}^{n} \lambda_{i}=$ 1 there exists $i_{0} \in\{1,2, \ldots, n\}$ such that $F\left(\sum_{i=1}^{n} \lambda_{i} x_{i}\right) \subset F\left(x_{i_{0}}\right)+C$ and $C$-quasiconvex-like if $-F$ is $C$-quasiconcave-like.

Remark 2.5 $C$-concave and $C$-quasiconcave-like are two different notions which cannot deduce from each other. For example, let $X=[0,1], R_{+}^{2}=[0,+\infty) \times[0,+\infty)$, vector valued function $f=\left(f_{1}, f_{2}\right)=(x,-x), g=\left(g_{1}, g_{2}\right)=\left(x^{2}, x^{2}\right)$. It is easy to prove that $f$ is $R_{+}^{2}$-concave but $f$ is not $R_{+}^{2}$-quasiconcave-like, inverse $g$ is $R_{+}^{2}$-quasiconcave-like but is not $R_{+}^{2}$-concave.

\section{Existence for weakly Ky Fan's points of set-valued mappings}

Lemma 3.1 (see [12]) Let $X$ be a nonempty subset of a Hausdorff topological vector space $E, F: X \rightarrow 2^{X}$ be a set-valued mapping. For each $x \in X, F(x)$ is closed, and there exists some $x_{0} \in X$ such that $F\left(x_{0}\right)$ is compact. If $\operatorname{co}\left\{x_{1}, x_{2}, \ldots, x_{n}\right\} \subset \bigcup_{i=1}^{n} F\left(x_{i}\right)$, where $\operatorname{co}\left\{x_{1}, x_{2}, \ldots, x_{n}\right\}$ is the convex hull of $\left\{x_{1}, x_{2}, \ldots, x_{n}\right\}$, then $\bigcap_{x \in X} F(x) \neq \emptyset$. 
Theorem 3.2 Let X be a nonempty convex compact subset of a Hausdorff topological vector space $E, C$ is a closed, convex, pointed cone with int $C \neq \emptyset$. If $\varphi: X \times X \rightarrow 2^{E}$ satisfies the following conditions:

(1) $\varphi(x, x) \not \subset$ int $C$ for all $x \in X$,

(2) for each fixed $y \in X, x \rightarrow \varphi(x, y)$ is $C$-quasiconcave-like,

then there exists $y^{*} \in X$ such that for each $x \in X$ and a net $\left\{y^{\alpha}\right\}$ with $\left\{y^{\alpha}\right\} \rightarrow y^{*}, \varphi\left(x, y^{\alpha}\right) \not \subset$ int $C$ for any $\alpha \in D$ (i.e., for each $x \in X$ and a neighborhood $N\left(y^{\prime \prime}\right)$ of $y^{\prime \prime}$, there exists a net $\left\{y^{\alpha}\right\} \in N\left(y^{*}\right)$ such that $\varphi\left(x, y^{\alpha}\right) \not \subset$ int $\left.C\right)$.

Proof Define a set-valued mapping $F: X \rightarrow 2^{X}$ as follows:

$$
F(x)=\{y \in X \mid \varphi(x, y) \not \subset \operatorname{int} C\}, \quad \forall x \in X .
$$

By (1), we can easily know that $F(x) \neq \emptyset$ for each $x \in X$. Next, we prove that for each $\left\{x_{1}, x_{2}, \ldots, x_{n}\right\} \subset X, c o\left\{x_{1}, x_{2}, \ldots, x_{n}\right\} \subset \bigcup_{i=1}^{n} F\left(x_{i}\right)(*)$. Suppose $(*)$ is not true, then there exist some $\left\{x_{1}, x_{2}, \ldots, x_{n}\right\} \subset X$ and $t_{i} \in[0,1], \sum_{i=1}^{n} t_{i}=1$ such that $x=\sum_{i=1}^{n} t_{i} x_{i} \notin \bigcup_{i=1}^{n} F\left(x_{i}\right)$. By the definition of $F(x)$, we can know that $\varphi\left(x_{i}, x\right) \subset$ int $C$ for each $i=1,2, \ldots, n$. By Theorem 3.2(2), Remark 2.3, and Definition 2.4(2), we can obtain that

$$
\varphi(x, x)=\varphi\left(\sum_{i=1}^{n} t_{i} x_{i}, x\right) \subset \varphi\left(x_{i_{0}}, x\right)+C \subset \operatorname{int} C+C \subset \operatorname{int} C,
$$

which contradicts the condition (1), thus $\operatorname{co}\left\{x_{1}, x_{2}, \ldots, x_{n}\right\} \subset \bigcup_{i=1}^{n} F\left(x_{i}\right)$ for each $\left\{x_{1}, x_{2}\right.$, $\left.\ldots, x_{n}\right\} \subset X$. Define a set-valued mapping $\mathrm{Cl}(F): X \rightarrow 2^{X}$ as follows,

$$
\mathrm{Cl}(F(x))=\mathrm{Cl}\{y \in X \mid \varphi(x, y) \not \subset \operatorname{int} C\}, \quad \forall x \in X
$$

where $\mathrm{Cl}(F(x))$ denotes the closure of $F(x)$. Clearly, for each $x \in X, \mathrm{Cl}(F(x)) \subset X, X$ is compact, so $\mathrm{Cl}(F(x))$ is compact. $\mathrm{By} F(x) \subset \mathrm{Cl}(F(x))$ and $(*)$, we know that $\mathrm{Cl}(F): X \rightarrow 2^{X}$ also satisfies (*), thus by Lemma 3.1 we have $\bigcap_{x \in X} \mathrm{Cl}(F(x)) \neq \emptyset$. Take $y^{*} \in \bigcap_{x \in X} \mathrm{Cl}(F(x))$, then $y^{*} \in \mathrm{Cl}(F(x))$ for each $x \in X$. Therefore, there exists $y^{\prime \prime} \in X$, such that for each $x \in X$ and a net $\left\{y^{\alpha}\right\}$ with $\left\{y^{\alpha}\right\} \rightarrow y^{*}, \varphi\left(x, y^{\alpha}\right) \not \subset$ int $C$ for any $\alpha \in D$. The proof is finished.

Corollary 3.3 Let X be a nonempty convex compact subset of a Hausdorff topological vector space $E, C$ is a closed, convex, pointed cone with int $C \neq \emptyset$. If a vector-valued function $\varphi: X \times X \rightarrow H$ satisfies the following conditions:

(1) $\varphi(x, x) \notin$ int $C$ for all $x \in X$,

(2) for each fixed $y \in X, x \rightarrow \varphi(x, y)$ is C-quasiconcave-like,

then there exists $y^{*} \in X$ such that for each $x \in X$ and a net $\left\{y^{\alpha}\right\}$ with $\left\{y^{\alpha}\right\} \rightarrow y^{*}, \varphi\left(x, y^{\alpha}\right) \notin$ int $C$ for any $\alpha \in D$.

Proof In Theorem 3.2, let $\varphi(x, y) \equiv H, \forall x \in X, \forall y \in X$.

Corollary 3.4 Let $X$ be a nonempty convex compact subset of a Hausdorff topological vector space E. If a function $\varphi: X \times X \rightarrow \Re$ satisfies the following conditions:

(1) $\varphi(x, x) \leq 0$ for all $x \in X$,

(2) for each fixed $y \in X, x \rightarrow \varphi(x, y)$ is quasiconcave, 
then there exists $y^{\prime \prime} \in X$ such that for each $x \in X$ and a net $\left\{y^{\alpha}\right\}$ with $\left\{y^{\alpha}\right\} \rightarrow y^{*}, \varphi\left(x, y^{\alpha}\right) \leq 0$ for any $\alpha \in D$.

Proof In Corollary 3.3, let $H=\Re, C=[0,+\infty)$.

Remark 3.5 From the proof process of Theorem 3.2, we can easily extend it to the case in which $X$ is not compact.

Theorem 3.6 Let $X$ be a nonempty convex subset of a Hausdorff topological vector space $E, C$ is a closed, convex, pointed cone with int $C \neq \emptyset$. If $\varphi: X \times X \rightarrow 2^{H}$ satisfies the following conditions:

(1) $\varphi(x, x) \not \subset$ int $C$ for all $x \in X$,

(2) for each fixed $y \in X, x \rightarrow \varphi(x, y)$ is $C$-quasiconcave-like,

(3) $\mathrm{Cl}\left(F\left(x_{0}\right)\right)=\mathrm{Cl}\left\{y \in X \mid \varphi\left(x_{0}, y\right) \not \subset\right.$ int $\left.C\right\}$ is compact,

then there exists $y^{*} \in X$ such that for each $x \in X$ and a net $\left\{y^{\alpha}\right\}$ with $\left\{y^{\alpha}\right\} \rightarrow y^{*}, \varphi\left(x, y^{\alpha}\right) \not \subset$ $\operatorname{int} C$ for any $\alpha \in D$.

Proof Define a set-valued mapping $F: X \rightarrow 2^{X}$ as follows:

$$
F(x)=\{y \in X \mid \varphi(x, y) \not \subset \operatorname{int} C\}, \quad \forall x \in X
$$

From the proof of Theorem 3.2, we can know that for each $\left\{x_{1}, x_{2}, \ldots, x_{n}\right\} \subset X, \operatorname{co}\left\{x_{1}, x_{2}\right.$, $\left.\ldots, x_{n}\right\} \subset \bigcup_{i=1}^{n} F\left(x_{i}\right)(*)$.

Define a set-valued mapping $\mathrm{Cl}(F): X \rightarrow 2^{X}$ as follows:

$$
\mathrm{Cl}(F(x))=\mathrm{Cl}\{y \in X \mid \varphi(x, y) \not \subset \operatorname{int} C\}, \quad \forall x \in X,
$$

where $\mathrm{Cl}(F(x))$ denotes the closure of $F(x)$. Clearly, for each $x \in X, \mathrm{Cl}(F(x))$ is closed. By Theorem 3.6(3), there exists $x_{0}$ such that $\mathrm{Cl}\left(F\left(x_{0}\right)\right)=\mathrm{Cl}\left\{y \in X \mid \varphi\left(x_{0}, y\right) \not \subset \operatorname{int} C\right\}$ is compact. Thus the conditions of Lemma 3.1 are satisfied. So we have $\bigcap_{x \in X} \mathrm{Cl}(F(x)) \neq \emptyset$. Take $y^{*} \in \bigcap_{x \in X} \mathrm{Cl}(F(x))$, then $y^{*} \in \mathrm{Cl}(F(x))$ for each $x \in X$. Therefore, there exists $y^{*} \in X$, such that for each $x \in X$ and a net $\left\{y^{\alpha}\right\}$ with $\left\{y^{\alpha}\right\} \rightarrow y^{\prime \prime}, \varphi\left(x, y^{\alpha}\right) \not \subset$ int $C$ for any $\alpha \in D$. The proof is finished.

In the same way, Corollary 3.3 and Corollary 3.4 can be promoted respectively as follows.

Corollary 3.7 Let $X$ be a nonempty convex subset of a Hausdorff topological vector space $E, C$ is a closed, convex, pointed cone with int $C \neq \emptyset$. If a vector-valued function $\varphi: X \times X \rightarrow$ $H$ satisfies the following conditions:

(1) $\varphi(x, x) \notin \operatorname{int} C$ for all $x \in X$,

(2) for each fixed $y \in X, x \rightarrow \varphi(x, y)$ is $C$-quasiconcave-like,

(3) $\mathrm{Cl}\left(F\left(x_{0}\right)\right)=\mathrm{Cl}\left\{y \in X \mid \varphi\left(x_{0}, y\right) \in \operatorname{int} C\right\}$ is compact,

then there exists $y^{*} \in X$ such that for each $x \in X$ and a net $\left\{y^{\alpha}\right\}$ with $\left\{y^{\alpha}\right\} \rightarrow y^{*}, \varphi\left(x, y^{\alpha}\right) \notin$ $\operatorname{int} C$ for any $\alpha \in D$.

Corollary 3.8 Let $X$ be a nonempty convex compact subset of a Hausdorff topological vector space E. If a function $\varphi: X \times X \rightarrow \Re$ satisfies the following conditions: 
(1) $\varphi(x, x) \leq 0$ for all $x \in X$,

(2) for each fixed $y \in X, x \rightarrow \varphi(x, y)$ is quasiconcave,

(3) $\mathrm{Cl}\left(F\left(x_{0}\right)\right)=\mathrm{Cl}\left\{y \in X \mid \varphi\left(x_{0}, y\right) \leq 0\right\}$ is compact,

then there exists $y^{\prime \prime} \in X$ such that for each $x \in X$ and any net $\left\{y^{\alpha}\right\}$ of $F(x)$ with $\left\{y^{\alpha}\right\} \rightarrow y^{\prime \prime}$, $\varphi\left(x, y^{\alpha}\right) \leq 0$ for any $\alpha \in D$.

Remark 3.9 By Remark 2.5, we know that $C$-concave and $C$-quasiconcave-like are two different notions which cannot deduce from each other. Then Theorem 3.2, Theorem 3.6 can easily extend the case in which for each fixed $y \in X, x \rightarrow \varphi(x, y)$ is $C$-concave in a similar way.

Remark 3.10 We call such points $y$ " the weakly Ky Fan's points in Theorem 3.2, Theorem 3.6. It is obvious that Ky Fan's points must be weakly Ky Fan's points, inverse is not true.

\section{Generic stability of the set for weakly Ky Fan's points of set-valued mappings}

In this section, we first give some lemmas and concepts, then we study the generic stability of the set for weakly Ky Fan's points for set-valued mappings.

Let $X$ be a nonempty convex compact subset of a Banach space $E$ with norm $\|\cdot\|, C$ be a closed, convex, pointed cone with int $C \neq \emptyset, K(E)$ be the set of all nonempty compact subsets of $E . M_{1}=\{\varphi: X \times X \rightarrow K(E) \mid \varphi$ satisfies the conditions (1), (2) in Theorem 3.2 $\}$.

$\forall \varphi_{1}, \varphi_{2} \in M_{1}$, define

$$
\rho\left(\varphi_{1}, \varphi_{2}\right)=\operatorname{Sup}_{(x, y) \in X \times X} h\left(\varphi_{1}(x, y), \varphi_{2}(x, y)\right),
$$

where $h\left(\varphi_{1}(x, y), \varphi_{2}(x, y)\right)$ denotes the Hausdorff distance between $\varphi_{1}(x, y)$ and $\varphi_{2}(x, y)$ on $X \times X$.

Clearly $\left(M_{1}, \rho\right)$ is a metric space, $(K(E), h)$ is complete metric space (see [11]). For any $\varphi \in M_{1}$, by Theorem 3.2, there exists $y^{*}$ a weakly Ky Fan's point of set-valued mappings. Let $F(\varphi)$ be the set of all weakly Ky Fan's points of $\varphi$, then $F(\varphi) \neq \emptyset$, and thus define a set-valued mapping from $M_{1}$ into $X, F: M_{1} \rightarrow 2^{X}$, where $F(\varphi)=\{y \in X \mid$ for each $x \in$ $X$ and a net $\left\{y^{\alpha}\right\}$ with $\left\{y^{\alpha}\right\} \rightarrow y$ we have $\varphi\left(x, y^{\alpha}\right) \not \subset$ int $C$ for any $\left.\alpha \in D\right\}$.

Next, we give some important lemmas in proving the generic stability of weakly Ky Fan's points for set-valued mappings.

Lemma 4.1 (see [13]) Let $X$ be a complete metric space, $Y$ is a metric space, $F: X \rightarrow 2^{Y}$ is an usco mapping. Then there is a dense $G_{\delta}$ subset $Q$ of $X$ such that $F$ is lower semicontinuous on $Q$.

Lemma 4.2 (see [11]) Let $X$ and $Y$ be two topological spaces with $Y$ is compact. If $F$ is a closed set-valued mapping from $X$ to $Y$, then $F$ is upper semi-continuous.

Lemma $4.3\left(M_{1}, \rho\right)$ is a complete metric space.

Proof Let $\left\{\varphi_{n}\right\}_{n=1}^{\infty}$ be any Cauchy sequence in $M_{1}$, then for any $\varepsilon>0$, there exists $N$ such that $\rho\left(\varphi_{n}, \varphi_{m}\right)$ for any $n, m \geq N$, i.e., $\operatorname{Sup}_{(x, y) \in X \times X} h\left(\varphi_{n}(x, y), \varphi_{m}(x, y)\right)<\varepsilon$ for any $n, m \geq N$. It follows that for each $(x, y) \in X \times X,\left\{\varphi_{n}(x, y)\right\}_{n=1}^{\infty}$ is a Cauchy sequence in $K(E)$. Since 
$K(E)$ is a complete metric space, there exists a compact set $\varphi(x, y) \in K(E)$ such that $h\left(\varphi_{n}(x, y), \varphi(x, y)\right) \leq \varepsilon(*)$ for any $(x, y) \in X \times X$. Next, we prove that $\varphi \in M_{1}$.

By $(*)$, we can obtain $\varphi_{n}(x, y) \subset U(\varphi(x, y), \varepsilon)$ and $\varphi(x, y) \subset U\left(\varphi_{n}(x, y), \varepsilon\right)$ for any $n \geq$ $N$, then we can obtain that $\varphi\left(\sum_{i=1}^{n} \lambda_{i} x_{i}, y\right) \subset U\left(\varphi_{n}\left(\sum_{i=1}^{n} \lambda_{i} x_{i}, y\right), \varepsilon\right)$. As $\varphi_{n} \in M_{1}$, and $x \rightarrow \varphi_{n}(x, y)$ is $C$-quasiconcave-like, we have $\varphi_{n}\left(\sum_{i=1}^{n} \lambda_{i} x_{i}, y\right) \subset \varphi_{n}\left(x_{i_{0}}, y\right)+C$ where $i_{0} \in$ $\{1, \ldots, n\}$. Thus we have $\varphi\left(\sum_{i=1}^{n} \lambda_{i} x_{i}, y\right) \subset U\left(\varphi_{n}\left(x_{i_{0}}, y\right)+C, \varepsilon\right) \subset U\left(\varphi\left(x_{i_{0}}, y\right)+C, 2 \varepsilon\right)$. Since $\varepsilon$ is arbitrary, $\varphi\left(\sum_{i=1}^{n} \lambda_{i} x_{i}, y\right) \subset \varphi\left(x_{i_{0}}, y\right)+C$, then $x \rightarrow \varphi(x, y)$ is $C$-quasiconcave-like. Now we suppose that $\varphi(x, x) \subset$ int $C$, then by $(*)$ we have $\varphi_{n}(x, x) \subset U(\varphi(x, x), \varepsilon)$. Since $\varepsilon$ is arbitrary, we can obtain that $\varphi_{n}(x, x) \subset \varphi(x, x)$, then we have $\varphi_{n}(x, x) \subset \varphi(x, x) \subset$ int $C$ which contradicts the assumption that $\varphi_{n}(x, x) \not \subset$ int $C$. Thus $\varphi(x, x) \not \subset$ int $C$. Hence, $\varphi \in M_{1},\left(M_{1}, \rho\right)$ is a complete metric space.

Lemma 4.4 $F: M_{1} \rightarrow 2^{X}$ is a usco mapping.

Proof Since $X$ is compact, by Lemma 4.2, it suffices to show that $F$ is a closed mapping, i.e., if for any $\varphi_{n} \in M_{1}, \varphi_{n} \rightarrow \varphi \in M_{1}, \forall z_{n} \in F\left(\varphi_{n}\right), z_{n} \rightarrow z$, then $z \in F(\varphi)$.

By $z_{n} \in F\left(\varphi_{n}\right)$, there exists a net $y^{\alpha_{n}} \rightarrow z_{n}$ and $\varphi_{n}\left(x, y^{\alpha_{n}}\right) \not \subset$ int $C$ for any $\alpha \in D$. Next, we suppose that $z \notin F(\varphi)$. Then there exists some $x$, and for each $y^{\alpha_{n}} \rightarrow z$, we have $\varphi\left(x, y^{\alpha_{n}}\right) \subset$ int $C$. As $\varphi_{n} \rightarrow \varphi$, we have $\varphi_{n}\left(x, y^{\alpha_{n}}\right) \subset U\left(\varphi\left(x, y^{\alpha_{n}}\right), \varepsilon\right)$ when $n \geq N$. Since $\varepsilon$ is arbitrary, we can obtain that $\varphi_{n}\left(x, y^{\alpha_{n}}\right) \subset \varphi\left(x, y^{\alpha_{n}}\right) \subset \operatorname{int} C$ which contradicts the assumption that $\varphi_{n}\left(x, y^{\alpha_{n}}\right) \not \subset$ int $C$. Thus, $z \in F(\varphi)$, i.e. $F$ is a closed mapping. Therefore, by Lemma 4.2, $F: M_{1} \rightarrow 2^{X}$ is a usco mapping.

Definition 4.5 Let $\varphi \in M_{1}$ (1) $y^{*} \in F(\varphi)$ is essential if for any $\varepsilon>0$, there exists $\delta>0$ such that for each $\varphi^{\prime} \in M_{1}$ with $\rho\left(\varphi, \varphi^{\prime}\right)<\delta$, there exists $y^{\prime} \in F\left(\varphi^{\prime}\right)$ with $\left\|y-y^{\prime}\right\|<\varepsilon$. (2) $\varphi$ is essential if every $y \in F(\varphi)$ is essential.

By Definition 2.1(2) and Definition 4.5, it is easy to obtain the following results.

Lemma 4.6 $\varphi$ is essential if and only if the set-valued mapping $F$ is lower semicontinuous on $\varphi$.

Theorem 4.7 There exists a dense $G_{\delta}$ subset $Q$ of $M_{1}$ such that each $\varphi \in Q$, $\varphi$ is essential.

Proof By Lemma 4.4, $F: M_{1} \rightarrow 2^{X}$ is a usco mapping. By Lemma 4.1, there exists a dense $G_{\delta}$ subset $Q$ such that each $\varphi \in Q, \varphi$ is lower semicontinuous on $Q$. By Lemma 4.6, for each $\varphi \in Q, \varphi$ is essential.

Remark 4.8 (1) Let $\varphi \in Q$. By Lemma 4.4 and Lemma 4.6, $F$ is continuous on $Q$. Then for any $\varepsilon>0$, there exists $\delta>0$ such that for any $\varphi^{\prime} \in M$, with $\rho\left(\varphi, \varphi^{\prime}\right)<\delta, h\left(F(\varphi), F\left(\varphi^{\prime}\right)\right)<\varepsilon$. Thus $\varphi$ is stable.

(2) Since $Q$ is a dense residual subset, it is the second category set, therefore most of $\varphi \in M_{1}$ have stable solution sets in the sense of Baire category.

Theorem 4.9 If $\varphi \in M_{1}$ is such that $F(\varphi)$ is a singleton set, then $\varphi$ is essential.

Proof For any open set $G$ of $X, F(\varphi) \cap G \neq \emptyset$, by $F(\varphi)=\{y\}$, then $y \in G$, and $G \supset F(\varphi)$. By Lemma 4.4, $F: M_{1} \rightarrow 2^{X}$ is upper semicontinuous. There exists an open neighborhood 
$O(\varphi)$ of $\varphi$ such that $G \supset F\left(\varphi^{\prime}\right)$ for any $\varphi^{\prime} \in O(\varphi)$, thus $G \cap F\left(\varphi^{\prime}\right) \neq \emptyset$, then $F$ is lower semicontinuous on $\varphi$. By Lemma 4.6, $\varphi$ must be essential.

\section{Competing interests}

The authors declare that they have no competing interests.

\section{Authors' contributions}

WSJ and SWX carried out the design of the study and performed the analysis. JHH and YLY participated in its design and coordination. All authors read and approved the final manuscript.

\section{Author details}

${ }^{1}$ College of Computer Science and Information, Guizhou University, Guiyang, Guizhou 550025, P.R. China. ${ }^{2}$ College of Science, Guizhou University, Guiyang, Guizhou 550025, P.R. China. ${ }^{3}$ College of Technology, Guizhou University, Guiyang, Guizhou 55004, P.R. China.

\section{Acknowledgements}

This work is supported by National Natural Science Foundation of China (11161008), Doctoral Program Fund for Ministry of Education (20115201110002) and Natural Science Fund of Guizhou Province (20122139).

Received: 4 March 2012 Accepted: 23 August 2012 Published: 7 September 2012

\section{References}

1. Fan, K: A minimax inequality and its applications. In: Inequality. Academic Press, New York (1972)

2. Lin, B, Simons, S: Nonlinear and Convex Analysis. Dekker, New York (1987)

3. Tan, KK, Yu, J, Yuan, XZ: The stability of Ky Fan's points. Proc. Am. Math. Soc. 123, 1511-1519 (1995)

4. Yu, J, Yuan, XZ: The study of Pareto equilibria for multiobjective games by fixed point and Ky Fan minimax inequality methods. Comput. Math. Appl. 35, 17-24 (1998)

5. Luo, Q: The essential component of the solution set for vector equilibrium problems. J. Glob. Optim. 34, 589-595 (2006)

6. Yang, H, Yu, J: Essential component of the weakly Pareto Nash equilibrium points. Appl. Math. Lett. 15, 553-556 (2002)

7. Yu, J, Yang, H, Yu, C: Well-posed Ky Fan's point, quasi-variational inequality and Nash equilibrium problems. Nonlinear Anal. TMA 66, 777-790 (2007)

8. Yu, J, Xiang, SW: On essential components of the set of Nash equilibrium points. Nonlinear Anal. TMA 38, 259-264 (1999)

9. Zhou, YH, Xiang, SW, Yang, H: Stability of solutions for Ky Fan's section theorem with some applications. Nonlinear Anal. TMA 62, 1127-1136 (2005)

10. Aubin, JP, Ekeland, I: Applied Nonlinear Analysis. Wiley, New York (1984)

11. Klein, E, Thompson, AC: Theory of Correspondence. Wiley, New York (1984)

12. Fan, K: A generalization of Tychonoff's fixed point theorem. Math. Ann. 142, 305-310 (1961)

13. $Y u$, J: Essential equilibria of $n$-person noncooperative games. J. Math. Econ. 31, 361-372 (1999)

doi:10.1186/1029-242X-2012-199

Cite this article as: Jia et al.: The existence and stability for weakly Ky Fan's points of set-valued mappings. Journal of Inequalities and Applications 2012 2012:199.

\section{Submit your manuscript to a SpringerOpen ${ }^{\circ}$ journal and benefit from:}

$\rightarrow$ Convenient online submission

Rigorous peer review

- Immediate publication on acceptance

- Open access: articles freely available online

- High visibility within the field

- Retaining the copyright to your article 